\title{
Data Sharing Through Query Translation in Autonomous Sources
}

\author{
Anastasios Kementsietsidis \\ Marcelo Arenas \\ Dept. of Computer Science \\ University of Toronto \\ \{tasos, marenas\}@cs.toronto.edu
}

\begin{abstract}
We consider the problem of data sharing between autonomous data sources in an environment where constraints cannot be placed on the shared contents of sources. Our solutions rely on the use of mapping tables which define how data from different sources are associated. In this setting, the answer to a local query, that is, a query posed against the schema of a single source, is augmented by retrieving related data from associated sources. This retrieval of data is achieved by translating, through mapping tables, the local query into a set of queries that are executed against the associated sources. We consider both sound translations (which only retrieve correct answers) and complete translations (which retrieve all correct answers, and no incorrect answers) and we present algorithms to compute such translations. Our solutions are implemented and tested experimentally and we describe here our key findings.
\end{abstract}

\section{Introduction}

We consider the problem of data sharing between autonomous structured data sources. Such sources may use different schemas to structure their data. Furthermore, both the data and the schemas of the sources may overlap little, if at all. Still, data residing in the different sources may be closely associated.

Permission to copy without fee all or part of this material is granted provided that the copies are not made or distributed for direct commercial advantage, the VLDB copyright notice and the title of the publication and its date appear, and notice is given that copying is by permission of the Very Large Data Base Endowment. To copy otherwise, or to republish, requires a fee and/or special permission from the Endowment.

Proceedings of the 30th VLDB Conference, Toronto, Canada, 2004
As an example, consider the domain of biological data sources. Different biological data sources can store inherently different data which range from data for genes or proteins to data for genetic diseases. Nevertheless, these diverse data sets are closely associated since genes encode for proteins and are related to genetic diseases.

How can we share data in such a setting? In the domain of biological sources, we can imagine that a biologist who queries her local source for information on a particular protein, say $\mathrm{OPH}$, would like to retrieve, in addition to the local data, related information that is found in any number of networked sources including related genes that encode for protein $\mathrm{OPH}$ and genetic diseases related to these genes. To support such sharing of data, we must be able to translate the local query into the vocabulary of the other sources. This involves translating both the structure of the query to use the schema elements of the associated sources, but also the data itself. For example, associated sources may use synonyms of protein $\mathrm{OPH}$, such as $\mathrm{APH}$ and AARE, or distinct identifiers for the same gene.

Notice that the related data that each source returns may be very different and it is often not possible to make this data conform to the local schema. For conformance to be possible, the local database must have anticipated the structure of all possible answers to a query. Mappings are needed to fit these structures into the local schema. Such a solution may be undesirable for several reasons. First, we may not wish to change the local schema to accommodate data for which it was not designed. In our example, the query results may be returned to users and not stored locally, so it seems onerous to insist that even virtual local structures be predefined for receiving this data. Second, if any of the networked data sources change their schemas, the local database must, somehow, become aware of this and update its mappings. Otherwise, the translated 
queries may not be valid on the updated sources. Again, this is an onerous requirement.

Data sharing deals with the exchange of data between heterogeneous sources whose data need not be interdependent and may represent different real world domains. In keeping with the literature, we refer to such autonomous, heterogeneous sources as peers. Data sharing between peers differs from the well-studied problems of data integration [12] and data exchange [6]. The latter two problems use schema-level mappings to express the relationships between heterogeneous schemas. In data integration, these mappings are used, at run time, to conform the data of one source to the schema of another. In data exchange, the mappings are used to populate a target schema with the data of a source schema.

In this work, we consider how to translate queries in the absence of such restrictive schema-level mappings. We make use of a form of data-level mappings called mapping tables which we first introduced in [11]. In brief, a mapping table contains a set of data associations between data values in two peer databases. Our previous work showed how to automate the management of mapping tables by checking the consistency of the associations and by inferring new associations from existing ones. Our current work focuses on how to use mapping tables during query answering. Our main contributions are:

- We introduce the semantics of query answering in an environment of autonomous peers. The semantics relies on the translation of queries between the peers through the use of mapping tables.

- We introduce the notions of sound translations (which only retrieve correct answers) and complete translations (which retrieve all correct answers, and no incorrect answers) to characterize the relationship between translated queries.

- We extend the definition of mapping tables to store not only associations between data values, but also associations between pairs of translated queries. This common representation of different types of associations permits more systematic and robust solutions for managing the associations.

- We present an algorithm for computing complete translations and an algorithm for testing if a query is a sound translation of another. We use the latter algorithm, and our ability to store past translations in mapping tables, to determine if a query can be translated (partially or in full) by means of the stored translations.

This paper is organized as follows. We motivate our solutions in Section 2, while Section 3 describes the related work. Section 4 presents the semantics of query answering and introduces the notions of sound and complete translations. Section 5 presents the algorithms for computing such translations. Sec- tion 6 discusses our implementation while Section 7 presents the experimental results. We conclude in Section 8 with a summary of the work.

\section{Motivating example}

In what follows, we consider two biological databases, namely MedLine and PubMed [1]. A portion of their schemas and instances can be seen in Figures 1 (a) and (b), respectively. Both databases store similar information about articles, namely, an article identifier, some keywords, which refer to protein names mentioned in the article, and date of publication (PubMed stores the month $(\mathrm{pm})$ and year $(p y)$ of publication, while MedLine stores only the year). In spite of the similarities in their schemas, the databases use different vocabularies to describe articles. For one thing, the two databases use their own local identifiers. Furthermore, they often refer to the same protein by using different names. For instance, OPH in MedLine and APH in PubMed refer to the same protein. We can use mapping tables to represent how values from different vocabularies may correspond [11]. In the same figure, we show examples of such tables. Mapping table keyword2kw associates keywords from MedLine to keywords in the PubMed relation. Notice that not all keywords from MedLine are mapped, that is, the tables might be incomplete. Mapping table id2id stores the identifiers of articles that are mentioned in both databases. Finally, mapping table year2yr uses a variable in its single tuple to represent the identity function, i.e., that each year in the first database is mapped to itself in the second. We note that not all attributes need to be mapped through mapping tables. For example, no table involves attribute $p m$ of the PubMed relation.

Example 1 Assume that a user wants to retrieve all MedLine articles that mention protein $\mathrm{OPH}$. Then a query such as the following may be used:

$$
\begin{aligned}
\mathcal{Q}_{1} & \text { select } \\
& \text { from MedLine } \\
& \text { where keyword = "OPH" }
\end{aligned}
$$

What if this user also wants to retrieve all PubMed articles mentioning the same protein? Given that $A P H$ and AARE are synonyms of OPH, the following query may be used:

$$
\begin{aligned}
\mathcal{Q}_{2}: & \text { select } \\
& \text { from PubMed } \\
& \text { where }(k w=\text { "APH" OR } k w=\text { "AARE") }
\end{aligned}
$$

Mapping tables might provide us with sufficient information to automate the process of translating a query posed against one database to a query posed 


\begin{tabular}{|l|l|l|}
\hline article_id & keyword & year \\
\hline 20185348 & OPH & 2000 \\
96281126 & OPH & 1996 \\
87051725 & NGF receptor & 1986 \\
99455262 & CRAF1 & 1999 \\
99455262 & TNF receptor & 1999 \\
\hline
\end{tabular}

(a) MedLine relation instance

\begin{tabular}{|l|l|l|l|}
\hline paper_id & $k w$ & $p y$ & $p m$ \\
\hline 10719179 & APH & 2000 & March \\
8724851 & AARE & 1996 & February \\
9915784 & p75 ICD & 1999 & January \\
10944856 & Sialidase 1 & 2000 & July \\
\hline
\end{tabular}

(b) PubMed relation instance

\begin{tabular}{|c||c|}
\hline keyword & $k w$ \\
\hline OPH & APH \\
OPH & AARE \\
NGF receptor & p75 ICD \\
G9 sialidase & Sialidase 1 \\
\hline
\end{tabular}

(c) Table keyword2kw

\begin{tabular}{|c|c|}
\hline article_id & paper_id \\
\hline 20185348 & 10719179 \\
\hline \multicolumn{2}{|c|}{ (d) Table id2id } \\
\hline year & py \\
\hline $\mathcal{X}$ & $\overline{\mathcal{X}}$ \\
\hline
\end{tabular}

(e) Table year2py
Figure 1: Instances and Mapping tables

against another, where both queries retrieve related data. The details of how this is achieved, and under which circumstances such a translation is possible, is the main focus of this work.

Notice that the retrieved data do not conform to the same schema. Even in this simple scenario, where the schemas are rather homogeneous, we cannot merge the results due to the difference in vocabularies. In general, we will not know before-hand what data we are going to retrieve and in what format. We expect that even the types of the retrieved data may differ significantly. For example, our biological scenario includes not only information about protein articles but also data about genes and diseases. One of the objectives of this work is to deal with this heterogeneity in the retrieved results.

Example 2 Continuing with our example, assume now that the user decides to execute query $\mathcal{Q}_{3}$ which retrieves PubMed articles mentioning protein APH:

$$
\begin{aligned}
\mathcal{Q}_{3} & \text { select } \\
& \text { from PubMed } \\
& \text { where } k w=" A P H "
\end{aligned}
$$

Intuitively, query $\mathcal{Q}_{3}$ satisfies the initial user selection requirements since it returns PubMed articles mentioning protein OPH. However, it does not retrieve all such articles. So, query $\mathcal{Q}_{3}$ is incomplete, compared to $\mathcal{Q}_{2}$. Nevertheless, neither query $\mathcal{Q}_{2}$ nor query $\mathcal{Q}_{3}$ return any incorrect answers, i.e., articles not mentioning protein $\mathrm{OPH}$.
Notions such as correctness (soundness) and completeness of query translations are formalized in the next sections. Soundness is a property that every translation must satisfy, however, executing queries that are incomplete is often sufficient. For one thing, users are often satisfied with incomplete answers if complete answers are overwhelming. We may also be able to cache the results from sound queries to deliver some answers faster. From a systems point of view, we show that significant savings in computation time can be achieved by reusing sound queries.

Our last remark concerns our representation of queries. Mapping tables allow us to store, as part of our database, the associations of values between different peers [11]. Motivated by the same rational, we offer here a similar representation for associating queries and their translations. This uniform representation allows us to develop a common set of tools to manage both data and query associations.

\section{Related work}

Our previous work on mapping tables focuses on the management of the tables and how these can be used for value-based lookups [11]. Thus, it does not consider structured queries. In the context of peer-topeer systems, advanced query mechanisms have been proposed by Harren et al [8] and Huebsch et al [9]. The latter work proposes structured query answering in an architecture that can scale to accommodate a large number of peers. However, peers must agree to support a common schema. Our work does not consider scalability issues but addresses instead issues relating to the heterogeneity of peers.

In Piazza, associations between peers are expressed through either global-as-view (GAV) or local-as-view (LAV) schema-level mappings [7, 14]. Both types of mappings are considered while translating queries between different peers. Our solutions are complimentary to this work since our framework operates in the absence of schema-level mappings and the only mappings used are in the data-level and have the form of mapping tables. The main difference between the two approaches is that while their work assumes that the retrieved data can be made to conform to the schema of the peer where a query is initiated, we make no such assumption.

$\mathrm{Ng}$ et al [15] also deal with the translation of queries in a network of peers. Descriptive keywords are used to associate schema elements of different peers. Then, the translation of queries is performed using the associated elements. A limitation of the approach is the underlying assumption that the keywords are used consistently throughout the peer network. Thus, unlike our work, their solutions cannot handle differences in the vocabularies within the data values of the peers. 
The work of Chang and Garcia-Molina [5] also deals with the translation of queries between heterogeneous sources. There, syntactic rules are used to map selection predicates from one database to that of another. At first glance, mapping tables look like materializations of these syntactic rules. However, the two constructs operate under different assumptions. A syntactic rule that maps MedLine article identifiers to PubMed article identifiers assumes that for any identifier of the former we can compute an identifier of the latter. Thus, the query translation process relies on this assumption to translate a query from the former database to one in the latter without having to deal with the intricacies of the mappings at the data level, that is, the fact that mappings are often incomplete. Our work makes no such assumptions and our translation techniques deal with exactly these circumstances. We also use a uniform representation both for the rules, i.e., the mappings between data values and for the queries and the mappings between translated queries.

\section{Query semantics}

We assume that query execution in our peer network uses a gossiping mechanism. The process is initiated by the execution of a user-defined query locally in a peer. Then, the user-defined query is forwarded either as is or in some translated form to either all or to a selected number of acquaintances of the current peer. Then, the execute-and-forward step is repeated in each of the forwarded peers, causing in turn the further propagation of the query. The process terminates after a fixed number of propagations of the initial user-defined query has occurred.

In accordance with the above, we assume hereafter that each query is defined, in terms of syntax, with respect to the schema of a single peer. Our thesis is that for a user to issue a query, she need only be aware of the local schema she is using. Over this schema, we assume that the user poses queries that involve only the operations of selection, projection and join. Still, this is a significant extension of the value-based lookups supported thus far. In terms of execution, queries are classified into two categories. A local query, much like a query in a centralized system, is executed using only the data in the local peer. On the other hand, a global query uses the peer network to augment locally retrieved data with data that reside in other peers. We now formalize the above notions and explain the query semantics with emphasis on the semantics of global queries.

Consider a set $P=\left\{P_{1}, P_{2}, \ldots, P_{n}\right\}$ of $n$ peers. Assume that peer $P_{i}$ exposes a set of attributes $U_{i}(1 \leq i \leq n)$ and that $U_{i} \cap U_{j}=\emptyset(i \neq j$, $1 \leq i, j \leq n)$, and let $r_{i}$ be an instance of $P_{i}$. A local query $q$, hereafter just called a query, over a

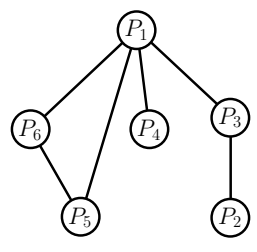

(a) The peer network

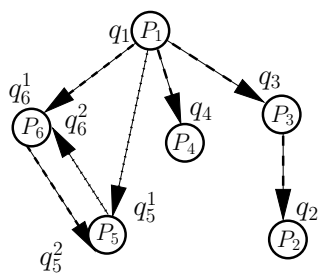

(b) The annotated network

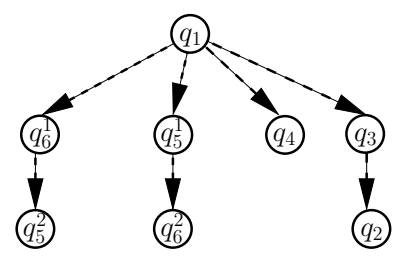

(c) The query dependency graph

Figure 2: A query dependency graph

peer $P_{i}(1 \leq i \leq n)$ is defined with respect to the schema of $P_{i}$. The result of $q$ is a relation over a set of attributes $V_{i}$, where $V_{i} \subseteq U_{i}$, whose content is the set of tuples $q\left(r_{i}\right)$. We denote by att $(q)$ the set of attributes $V_{i}$. A global query $q_{P}$ over the peers in $P$ is a set of queries $\left\{q_{1}^{1}, q_{1}^{2}, \ldots, q_{1}^{k_{1}}, \ldots, q_{n}^{1}, q_{n}^{2}, \ldots, q_{n}^{k_{n}}\right\}$, where query $q_{i}^{j}\left(1 \leq i \leq n\right.$ and $\left.1 \leq j \leq k_{i}\right)$ is over the schema of peer $P_{i}$ and $\operatorname{att}\left(q_{i}^{j}\right)=\operatorname{att}\left(q_{i}^{l}\right)$, for every $l \in\left[1, k_{i}\right]$. Each query in $q_{P}$ is called a component of $q_{P}$ and, conversely, $q_{P}$ is said to be comprised of the indicated set. The result of global query $q_{P}$ is a relation over the schema $R_{P}\left[V_{1} \cup V_{2} \cup \ldots \cup V_{n}\right]$, where $V_{i}=\operatorname{att}\left(q_{i}^{1}\right)=\cdots=\operatorname{att}\left(q_{i}^{k_{i}}\right)(1 \leq i \leq n)$, whose content is the set of tuples in the outer-union of the union of the queries in each peer, that is, the outerunion of $\bigcup_{j=1}^{k_{1}} q_{1}^{j}\left(r_{1}\right), \bigcup_{j=1}^{k_{2}} q_{2}^{j}\left(r_{2}\right), \ldots, \bigcup_{j=1}^{k_{n}} q_{n}^{j}\left(r_{n}\right)$. Hence, we permit peers to return results with different schemas. We also propose not to merge results as merging semantics tend to be application specific. Given these definitions, each local query can be thought of as a trivial global query which is comprised of a single component query.

Example 3 The set $\left\{\mathcal{Q}_{1}, \mathcal{Q}_{2}\right\}$ of queries (from $E x$ ample 1) is a global query over the MedLine and PubMed peers. The execution of this global query is initiated by local query $\mathcal{Q}_{1}$. The result of this global query is a relation with attributes $\{$ article_id, keyword, year, paper_id, kw, py, pm\}, whose content is the outer-union of the relations obtained by applying queries $\mathcal{Q}_{1}$ and $\mathcal{Q}_{2}$ to the instances shown in Figures 1 (a) and (b), respectively.

We now examine the relationship between the component queries of a global query. For this, we introduce the notion of query dependencies. Intuitively, as queries are propagated in the system, a 
(directed) query dependency graph is induced. The nodes in this graph represent queries and there is an edge from query $q_{j}$ to query $q_{k}$ if query $q_{k}$ depends on query $q_{j}$. A query $q_{k}$ on peer $P_{k}$ is said to depend on query $q_{j}$ over peer $P_{j}$, denoted as $\operatorname{dep}\left(q_{k}\right)=q_{j}$, if peers $P_{j}$ and $P_{k}$ are acquainted and query $q_{k}$ has resulted from the propagation of query $q_{j}$ on peer $P_{j}$ to peer $P_{k}$. Special care is taken to avoid the creation of cycles in the induced graph. This happens if a query propagated by a peer $P_{k}$ is re-received by the same peer through one of its acquaintances. Tagging queries with the path of peers through which they are propagated allows the detection of such situations. In Figure 2(a) there is an example of a peer network that consists of six peers, i.e., $P=\left\{P_{1}\right.$, $\left.P_{2}, \ldots, P_{6}\right\}$ and there is an edge between two peers if they are acquainted. In Figure 2(b), we use an intermediate representation where each peer in the network is annotated with the component queries of global query $q_{P}=\left\{q_{1}, q_{2}, q_{3}, q_{4}, q_{5}^{1} q_{5}^{2}, q_{6}^{1}, q_{6}^{2}\right\}$ that it executes. The edges in this representation show the propagation of queries. Finally, Figure 2(c) shows the dependency graph of $q_{P}$. Notice that in some peers more than one query is executed. For example, in peer $P_{5}$ we have the execution of two queries, namely $q_{5}^{1}$ and $q_{5}^{2}$. Query $q_{5}^{1}$ results from the propagation of query $q_{1}$ from peer $P_{1}$ while query $q_{5}^{2}$ results from the propagation of query $q_{6}^{1}$ from peer $P_{6}$. We devote the next paragraphs examining how exactly the propagation, and possible translation, of queries is achieved between peers.

\subsection{Mapping tables}

We offer here an overview of mapping tables since they are the main vehicle used for query translation.

Consider two peers that expose relations with attributes $U$ and $V$, respectively. A mapping table is a relation over the attributes $X \cup Y$, where $X \subseteq U$ and $Y \subseteq V$ are non-empty sets of attributes from the two peers. For example, a mapping table from a set of attributes $X=\{k e y w o r d\}$ to a set of attributes $Y=\{k w\}$ is shown in Figure 1(c). A vertical double line is used to separate the two attributes sets.

To represent different semantics for mapping tables and values within them, the standard convention of using variables is followed. For instance, Figure 1(e) shows a mapping table containing variables. Every valuation of these variables gives a value of year that can be mapped to a value of $p y$. Since this mapping table contains the same variable in its two columns, every valuation is a tuple of the form $(a, a)$, where $a$ is a constant in the domains of year and $p y$. Thus, in this case variables offer a compact way of representing the identity mapping.

Mapping tables restrict the way in which information may be exchanged between peers, instead of restricting their contents. Let $r_{1}$ and $r_{2}$ be instances of peers $P_{1}$ and $P_{2}$, respectively, and $m$ be a mapping table from $X$ to $Y$, where $X$ and $Y$ are subsets of the set of attributes exposed by $P_{1}$ and $P_{2}$, respectively. Given a valuation $\rho$ of the variables of $m$, a value $x \in \pi_{X}(\rho(m))$ is associated with the set of values $\pi_{Y}\left(\sigma_{X=x}(\rho(m))\right)$ and, hence, each $t_{1} \in r_{1}$ such that $t_{1}[X]=x$ can be mapped only to the tuples $t_{2} \in r_{2}$ for which $t_{2}[Y] \in \pi_{Y}\left(\sigma_{X=x}(\rho(m))\right)$.

\subsection{Sound and complete translations}

We are interested in translating, through mapping tables, queries that involve the operations of projection, selection and join. In what follows, we consider how this is achieved in the presence of the latter two operators. Then, in Section 4.3 we show how to handle projections. This separation is possible since, as we show, the issues involved are orthogonal.

Consider peers $P_{1}$ and $P_{2}$ that expose attributes $U_{1}$ and $U_{2}$, respectively. To begin, we assume that a single mapping table $m$ exists with schema $M\left[U_{1} \cup\right.$ $U_{2}$ ] that associates values of $U_{1}$ to values of $U_{2}$. We relax this assumption later. Consider two queries $q_{1}$ and $q_{2}$ over peers $P_{1}$ and $P_{2}$, respectively, such that $\operatorname{dep}\left(q_{2}\right)=q_{1}$, that is, query $q_{2}$ resulted from the propagation and translation of query $q_{1}$. We claim that the nature of this translation should be such that $q_{2}$ retrieves from peer $P_{2}$ only the data that are related with those that could be retrieved from query $q_{1}$ in peer $P_{1}$. The exact relationship is determined by the set of mapping tables that exists between the two peers.

Definition 4 Let $q_{1}$ and $q_{2}$ be queries over peers $P_{1}$ and $P_{2}$, respectively, such that $q_{1}=\sigma_{E}\left(R_{1} \bowtie \cdots \bowtie\right.$ $\left.R_{k}\right)$, where $E$ is a conjunction of equality atoms and $R_{1}, \ldots, R_{k}$ are relations in $P_{1}$. Then $q_{2}$ is a sound translation of $q_{1}$ with respect to mapping table $m$, denoted by $q_{1} \stackrel{m}{\longmapsto} q_{2}$, if for every relation instance $r_{2}$ of $P_{2}$ and $t_{2} \in q_{2}\left(r_{2}\right)$, there exists a valuation $\rho$ of $m$ and a tuple $t \in \sigma_{E}(\rho(m))$ such that $\pi_{\text {att }\left(q_{2}\right)}(t)=t_{2}$.

We offer a few remarks on our definition. First, observe that in the definition query $q_{1}$ operates on mapping table $m$, instead of operating on some instance $r_{1}$ of $P_{1}$, while query $q_{2}$ operates on an instance $r_{2}$ of $P_{2}$. This is to allow for query $q_{2}$ to retrieve data from $r_{2}$ that could be mapped to some instance $r_{1}$ of $P_{1}$ through $m$, but that it is not necessary for the data retrieved from $q_{1}$. Second, note that since $m$ contains all the attributes mentioned in $R_{1}, \ldots, R_{k}$, in order to evaluate $q_{1}$ in the relation $\rho(m)$ we do not need to compute the join of $R_{1}, \ldots$, $R_{k}$, we just have to check the condition $E$.

Example 5 Consider the mapping table ML2PM shown in Figure 3. Since all variables in the ta- 


\begin{tabular}{|c|c|c||c|c|c|c|}
\hline article_id & keyword & year & paper_id & $k w$ & $p y$ & $p m$ \\
\hline $\mathcal{X}_{1}$ & OPH & $\mathcal{Y}_{1}$ & $\mathcal{X}_{2}$ & APH & $\mathcal{Y}_{2}$ & $\mathcal{Z}_{2}$ \\
$\mathcal{X}_{3}$ & OPH & $\mathcal{Y}_{3}$ & $\mathcal{X}_{4}$ & AARE & $\mathcal{Y}_{4}$ & $\mathcal{Z}_{4}$ \\
\hline
\end{tabular}

Figure 3: Mapping table ML2PM.

ble are distinct, the table essentially maps protein $O P H$ in MedLine to proteins APH and AARE in PubMed. Now, consider queries $\mathcal{Q}_{1}$ and $\mathcal{Q}_{2}$ from our motivating example (see Section 2). Query $\mathcal{Q}_{2}$ is a sound translation of query $\mathcal{Q}_{1}$ with respect to the table ML2PM. On the other hand, the following query is not a sound translation of query $\mathcal{Q}_{1}$.

$$
\begin{aligned}
\mathcal{Q}_{4} & \text { select } \\
& \text { from PubMed } \\
& \text { where } k w=" A P H " \text { OR } k w=" p 75 I C D "
\end{aligned}
$$

To see this, consider the PubMed relation instance in Figure 1(b). Its third tuple satisfies $\mathcal{Q}_{4}$ but it cannot be associated, through ML2PM, to any MedLine article retrieved by $\mathcal{Q}_{1}$.

Another observation is that the above definition is not symmetric. Also, note that sound translations are not unique.

Example 6 While our previous example shows that $\mathcal{Q}_{4}$ is not a sound translation of $\mathcal{Q}_{1}$, notice that $\mathcal{Q}_{1}$ is a sound translation of $\mathcal{Q}_{4}$, with respect to table PM2ML (which is the inverse of the ML2PM). Tuples retrieved by $\mathcal{Q}_{1}$, from every possible instance of MedLine, correspond to articles mentioning protein OPH which, through PM2ML, can be associated with PubMed articles mentioning protein APH. Concerning the uniqueness of sound translations, remember from our motivating example that both queries $\mathcal{Q}_{2}$ and $\mathcal{Q}_{3}$ are sound translations of query $\mathcal{Q}_{1}$.

Since one sound translation might retrieve more data than another, we consider next the notion of completeness. That is, whether there is a sound translation that retrieves from remote peers all possible sound data.

Definition 7 Given queries $q_{1}, q_{2}$ over peers $P_{1}$ and $P_{2}$, respectively, we say that $q_{2}$ is a complete translation of query $q_{1}$ with respect to mapping table $m$, if $q_{1} \stackrel{m}{\mapsto} q_{2}$ and for every query $q_{2}^{\prime}$ over $P_{2}$ such that $q_{1} \stackrel{m}{\mapsto} q_{2}^{\prime}$ and every instance $r_{2}$ of $P_{2}$, $q_{2}\left(r_{2}\right) \supseteq q_{2}^{\prime}\left(r_{2}\right)$.

Notice that, by definition, if two queries $q_{2}$ and $q_{2}^{\prime}$ are complete translations of a query $q_{1}$, then $q_{2}$ and $q_{2}^{\prime}$ are equivalent.

Example 8 Consider query $\mathcal{Q}_{1}$ from our motivating example and its sound translations, namely, $\mathcal{Q}_{2}$ and $\mathcal{Q}_{3}$. We claim, without providing a formal proof, that query $\mathcal{Q}_{2}$ is a complete translation of $\mathcal{Q}_{1}$, with respect to mapping table ML2PM.

We are now in a position to formally characterize the relationship between the component queries of a global query. Specifically, we require that for each pair $q_{i}, q_{j}$ of component queries such that $\operatorname{dep}\left(q_{j}\right)=$ $q_{i}$, query $q_{j}$ is a sound translation of query $q_{i}$.

Our definitions assume that a single mapping table maps all the attributes in the relations involved. We relax this assumption in Section 5.5. We also note that so far we have only considered the selection and join operators. We investigate the issues concerning the projection operator next.

\subsection{The projection operator}

Sound translations guarantee that only correct tuples are retrieved from remote peers. However, not all the attributes from the remote peers are necessarily of interest. The user has the ability, through the projection operator, to express what local attributes are of interest to her and, thus, we provide a similar mechanism for the data retrieved from remote peers.

Our solutions make use of attribute correspondences which associate attributes in different peers. Learning attribute correspondences is a main component of schema matchers [16]. An attribute correspondence for attributes requiring no data translation can be encoded by a simple mapping table with the identity mapping. This is the situation depicted in Figure 1(e). In general, a mapping table $m[X \cup Y]$ encodes, in addition to the set of data associations, an attribute correspondence between the set of attributes $X$ and $Y$.

Definition 9 Let $P$ and $P^{\prime}$ be peers exposing set of attributes $U$ and $U^{\prime}$, respectively, and $m[X \cup Y]$ be a mapping table such that $X \subseteq U$ and $Y \subseteq U^{\prime}$. Then, $m$ is relevant to a query $q$ over $P$, if $\operatorname{att}(q) \subseteq X$.

Hence, when translating a query $q$ that includes a projection on attributes att $(q)$ we make use of all the relevant mapping tables $m_{1}\left[X_{1} \cup Y_{1}\right], m_{2}\left[X_{2} \cup Y_{2}\right]$, $\ldots m_{k}\left[X_{k} \cup Y_{k}\right]$, and the translated query returns the union of all the $Y_{i}$ 's in these tables.

Example 10 Consider the query that retrieves from MedLine all the protein names mentioned in articles published in 1998:

$$
\begin{aligned}
\mathcal{Q}_{5}: & \text { select keyword } \\
& \text { from MedLine } \\
& \text { where year = "1998" }
\end{aligned}
$$

The complete translation of $\mathcal{Q}_{5}$, with respect to mapping table year2py shown in Figure 1(e), is: 


$$
\begin{aligned}
\mathcal{Q}_{6}: & \text { select } k w \\
& \text { from PubMed } \\
& \text { where py }=\text { "1998" }
\end{aligned}
$$

There are two points to make here. First, due to mapping table year2py, our projection is on the $k w$ attributes of the retrieved PubMed tuples. Second, it is possible that the latter query retrieves PubMed articles that violate mapping table keyword2kw shown in Figure 1(c). However, this is consistent with our query semantics since soundness is defined here only with respect to the year2py mapping table.

\section{Algorithms}

In general, queries are initially expressed in a query language (e.g., relational algebra, SQL) and are later transformed in some appropriate internal representation. Before we discuss the issue of querying, we need to fix these two parameters, i.e., the query language and the representation used.

We focus here on queries that are expressed in $S+J$ algebra. An $S+J$ query uses the operators of selection and join. The selection formula is positive, i.e., it has no negation and it consists of conjunctions and disjunctions of atoms of the form $(A=B)$ and $(A=a)$, where $A$ and $B$ are attribute names and $a$ is a constant. Note that projection is supported in our framework but is handled independently.

Common query representations include tableau, which is a tabular representation of a query which resembles a database instance, and query trees, which is a graph-like representation of a query [3]. In this work, the tabular representation is the preferred choice. One reason for this is uniformity. Notice that we already use a similar representation, namely, mapping tables, to address issues of heterogeneity among different peers. In the following paragraphs, we introduce T-queries which is a tabular representation of queries and we show that for each $S+J$ query we can have an equivalent T-query. Then, we show how T-queries can be used to test whether a query $q^{\prime}$ is a sound translation of query $q$. Finally, we show how T-queries can be used to compute sound and complete query translations.

\subsection{T-queries}

We start by defining T-queries over one relation. Thus, we only consider selections. We later show how our definitions are extended to consider queries over multiple relations, thus taking into account joins. The following paragraph presents the syntax and semantics of T-queries.

A T-query $q_{T}$ over relation schema $R[U]$ is a table $T$ with attributes $U$ where each variable appears in at most one row. Intuitively, one can think of each $t \in T$ as a tableau query whose corresponding tableau only has a single tuple. Then, $T$ represents a set of tableau queries. Given a T-query $q_{T}$ over schema $R$ and an instance $r$ of $R$, the result of executing $q_{T}$ on $r$, denoted as $q_{T}(r)$ is:

$q_{T}(r)=\{\rho(t) \mid \rho$ is a valuation of $t \in T$ and $\rho(t) \in r\}$.

Example 11 Consider the following query that returns all articles from PubMed mentioning proteins APH or p75 ICD:

$$
\begin{aligned}
\mathcal{Q}_{7}: & \text { select } \\
& \text { from PubMed } \\
& \text { where } k w=" A P H " \text { OR } k w=" p 75 I C D "
\end{aligned}
$$

Then, the corresponding T-query is shown below:

\begin{tabular}{|c|c|c|c|}
\hline paper_id & $k w$ & $p y$ & $p m$ \\
\hline $\mathcal{X}_{1}$ & $A P H$ & $\mathcal{Y}_{1}$ & $\mathcal{Z}_{1}$ \\
$\mathcal{X}_{2}$ & $p 75 I C D$ & $\mathcal{Y}_{2}$ & $\mathcal{Z}_{2}$ \\
\hline
\end{tabular}

Proposition 12 For any $S+$ query $q$ over a relation $R[U]$ there is an equivalent T-query $q_{T}$, and vice versa.

To construct query $q_{T}$ from $q$, first we have to transform $q$ into an equivalent query $q^{\prime}$ of the form $\sigma_{E}(R)$, where $E$ is in disjunctive normal form. T-query $q_{T}$ is of size linear in the size of $q^{\prime}$, which, in turn, has a size which is exponential, in general, with respect to the size of the initial query $q$. However, this is not a problem in practice since large selection formulas rarely occur.

We extend the definition of T-queries over multiple relations in the following way. Let $R=\left\{R_{1}\left[U_{1}\right]\right.$, $\left.R_{2}\left[U_{2}\right], \ldots, R_{n}\left[U_{n}\right]\right\}$ be a relational schema and $U$ be $\cup_{i=1}^{n} U_{i}$. A T-query $q_{T}$ over $R$ is a table $T$ with attributes $U$ where each variable appears in at most one row. In terms of semantics, consider a T-query $q_{T}$ over $R$ and an instance $r=\left\{r_{1}, r_{2}, \ldots, r_{n}\right\}$ of $R$. Then the result of executing $q_{T}$ on $r$, denoted by $q_{T}(r)$, is:

$$
\begin{aligned}
& q_{T}(r)=\{\rho(t) \mid \rho \text { is a valuation of } t \in T \\
& \text { and } \left.\rho(t) \in r_{1} \bowtie \cdots \bowtie r_{n}\right\} .
\end{aligned}
$$

Proposition 13 For any $S+J$ query $q$ over a relational schema $R$, there is an equivalent T-query $q_{T}$, and vice versa.

We conclude this subsection by presenting the notion of join between T-queries. This will play a central role in all the algorithms presented in the following subsections. Let $T_{1}$ and $T_{2}$ be the tables of T-queries $q_{T}^{1}$ and $q_{T}^{2}$ with attributes $U_{1}$ and $U_{2}$, respectively. Attribute sets $U_{1}$ and $U_{2}$ are not necessarily disjoint. Then $T_{1} \bowtie T_{2}$ is a T-query with attributes $U_{1} \cup U_{2}$ defined as follows. Recall that 
a substitution is a function that maps only variables to either variables or constants. For every $t_{1} \in T_{1}$ and $t_{2} \in T_{2}$, find substitutions $\theta_{1}$ and $\theta_{2}$ for the variables of $t_{1}$ and $t_{2}$, respectively, such that $\theta_{1}\left(t_{1}\left[U_{1} \cap U_{2}\right]\right)=\theta_{2}\left(t_{2}\left[U_{1} \cap U_{2}\right]\right)$. Furthermore, we require that for any other pair of substitutions $\theta_{1}^{\prime}$ and $\theta_{2}^{\prime}$ satisfying this condition, $\theta_{1}$ is as general as $\theta_{1}^{\prime}$ and $\theta_{2}$ is as general as $\theta_{2}^{\prime}$, that is, there exist substitutions $\gamma_{1}$ and $\gamma_{2}$ such that $\gamma_{1} \circ \theta_{1}=\theta_{1}^{\prime}$ and $\gamma_{2} \circ \theta_{2}=\theta_{2}^{\prime}$ (this corresponds to the notion of most general unifier used in logic programming [13]). If substitutions $\theta_{1}$ and $\theta_{2}$ exist, then add to $T_{1} \underset{\wedge}{\mathrm{var}} T_{2}$ a $U_{1} \cup U_{2}$-tuple $t$ defined as: $t\left[U_{1}\right]=\theta_{1}\left(t_{1}\right)$ and $t\left[U_{2}\right]=\theta_{2}\left(t_{2}\right)$.

Intuitively, given relation instances $r_{1}$ and $r_{2}$ over $U_{1}$ and $U_{2}$, respectively, the join of $T_{1}$ and $T_{2}$ gives us a new T-query $q_{T}$ such that:

$$
q_{T}\left(r_{1} \bowtie r_{2}\right)=q_{T}^{1}\left(r_{1}\right) \bowtie q_{T}^{2}\left(r_{2}\right) .
$$

\subsection{Algorithms for sound translations}

In the previous section, we introduced sound translations and we provided a definition through which we can test, given two queries $q$ and $q^{\prime}$ over peers $P$ and $P^{\prime}$, respectively, whether query $q^{\prime}$ is a sound translation of $q$ with respect to a mapping table $m$. The definition of sound translations, however, does not provide us with a practical way to do the testing. In the next paragraphs, we show that one of the benefits of representing queries as T-queries is that we are able to perform such a test efficiently.

In brief, the proposed algorithm accepts as input two queries $q$ and $q^{\prime}$ over peers $P$ and $P^{\prime}$, respectively, and a mapping table $m$ between the sets of attributes $U$ and $U^{\prime}$ exposed by these peers. Initially, it converts both $q$ and $q^{\prime}$ to their corresponding T-queries, say $q_{T}=T$ and $q_{T}^{\prime}=T^{\prime}$, respectively. Then the algorithm uses mapping table $m$ to constrain the association of query disjunctions. Formally, given $T, m$ and $T^{\prime}$, the algorithm constructs a T-query $q_{T}^{c}$ with attributes $\operatorname{att}\left(q^{\prime}\right)$ defined as $\pi_{\text {att }\left(q^{\prime}\right)}\left(T \stackrel{\text { var }}{\bowtie} \underset{\bowtie}{\operatorname{var}} T^{\prime}\right)$. We note that it is possible to perform this join since mapping tables and T-queries use the same syntax. In the last step, the algorithm checks whether $q_{T}^{c}$ is equivalent to $q_{T}^{\prime}$, that is, for every instance $r^{\prime}$ of peer $P^{\prime}$, $q_{T}^{c}\left(r^{\prime}\right)=q_{T}^{\prime}\left(r^{\prime}\right)$. To perform such a test we use an algorithm that checks for containment of union of conjunctives queries [17]. If $q_{T}^{c}$ is equivalent to $q_{T}^{\prime}$, then the algorithm outputs yes.

Theorem 14 The above algorithm outputs yes on input $\left(q, m, q^{\prime}\right)$ if and only if $q^{\prime}$ is a sound translation of $q$ with respect to mapping table $m$.

The following proposition is used in the proof of this theorem. It also shows that the previous al-

\begin{tabular}{|c|c|c||c|c|c|c|}
\hline article_id & keyword & year & paper_id & $k w$ & $p y$ & $p m$ \\
\hline $\mathcal{X}_{1}$ & OPH & 1998 & $\mathcal{X}_{2}$ & APH & 1998 & $\mathcal{Y}_{2}$ \\
$\mathcal{X}_{3}$ & OPH & 1998 & $\mathcal{X}_{4}$ & AARE & 1998 & $\mathcal{Y}_{4}$ \\
$\mathcal{X}_{5}$ & OPH & 2003 & $\mathcal{X}_{6}$ & APH & 2003 & $\mathcal{Y}_{6}$ \\
$\mathcal{X}_{7}$ & OPH & 2003 & $\mathcal{X}_{8}$ & AARE & 2003 & $\mathcal{Y}_{8}$ \\
\hline
\end{tabular}

Figure 4: Storing query translations

gorithm only needs to check whether $q_{T}^{\prime} \subseteq q_{T}^{c}$ to verify whether $q^{\prime}$ is a sound translation of $q$.

Proposition 15 The queries $q_{T}^{\prime}$ and $q_{T}^{c}$ computed by the above algorithm on input $\left(q, m, q^{\prime}\right)$ are such that $q_{T}^{c} \subseteq q_{T}^{\prime}$.

Finally, we establish the exact complexity of our problem.

Theorem 16 The problem of testing whether a query is a sound translation of another query is $\Pi_{2}^{p}$ complete.

\subsection{Computing complete translations}

Here we describe an algorithm that, given a query $q$ and a mapping table $m$, computes a query $q^{\prime}$ such that $q^{\prime}$ is a sound and complete translation of $q$ with respect to mapping table $m$. The algorithm extends the algorithm for testing sound translations. Let $P$ and $P^{\prime}$ be two peers that expose attributes $U$ and $U^{\prime}$, respectively, and assume that $q$ is a query over $P$ and $m$ is mapping table between the set of attributes $U$ and $U^{\prime}$. The algorithm begins by converting query $q$ to its corresponding T-query $q_{T}=T$. Then, it considers mapping table $m$ and computes T-query $q_{T}^{\prime}=\pi_{U^{\prime}}(T \stackrel{\text { var }}{\bowtie} m)$. Finally, the algorithm outputs the query $q^{\prime}$ represented by $q_{T}^{\prime}$. The following theorem shows that $q^{\prime}$ is a sound and complete translation of query $q$.

Theorem 17 Query $q^{\prime}$ computed by the above algorithm on input $(q, m)$ is a complete translation of $q$.

\subsection{Composing translations}

In this section, we show the benefits of using a common formalism for representing both data and query associations. In more detail, we show that the algorithms that were created for inference of mapping tables can be used to effectively perform query composition. Consider the point in time after a complete translation has been computed. This translation may be stored within a mapping table. An example is shown in Figure 4. The query on the left of the table retrieves MedLine articles that mention protein OPH and were published in 1998 or 2003. 
The query on the right represents a complete translation on PubMed. Notice that each tuple in the mapping table pairs a query with a sound translation of the query. Such storage permits us to reuse the data association inference algorithm of our earlier work [11] to compose query translations. Consider a path $\theta=P_{1}, P_{2}, \ldots, P_{n}$ of peers with a set of mapping tables $m_{i}$ storing data associations between peers $P_{i}$ and $P_{i+1}$, for $1 \leq i \leq n-1$. Now let $T_{i}$ be a mapping table containing pairs of sound query translations (that is, each tuple $\left(q, q^{\prime}\right)$ in the mapping table represents a T-query $q$ on $P_{i}$ and a sound translation $q^{\prime}$ of $q$ with respect to the mapping table $m_{i}$ ).

Let $m$ denote the mapping table that results from our inference algorithm [11] over the path $\theta$ and the set of mapping tables $m_{i}$. And let $T$ denote the mapping table that results from our inference algorithm over the path $\theta$ and the set of (query) mapping tables $T_{i}$. Then, each tuple $\left(q, q^{\prime}\right)$ in $T$ contains a Tquery $q^{\prime}$ that is a sound translation of $q$ with respect to the mapping table $m$.

\subsection{Using multiple mapping tables}

The algorithms presented in the previous sections assume the existence of a single mapping table that maps all the attributes in the relations involved. In real life, we expect that multiple mapping tables are provided and that some attributes are not mapped. In what follows, we investigate how to handle these two situations.

Assume that instead of a single mapping table $m$, we are given a set of mapping tables $M$ to use during the computation of a complete translation. The exact way in which these tables are combined can be either pre-specified or it can be left to the user. Here, we propose a technique for combining multiple tables automatically. The following example illustrates that combining all available tables during the computation of translations might yield counterintuitive translations.

Example 18 Consider query $\mathcal{Q}_{1}$ from our motivating example that retrieves articles from MedLine mentioning protein $\mathrm{OPH}$. Assume that instead of just using mapping table keyword2kw, in Figure 1(c), we consider both mapping tables keyword2kw and id2id to compute the translation. Furthermore, assume that every retrieved tuple from PubMed must be associated with a local OPH article with respect to both mapping tables. Then, the resulting translation is equivalent to the following query:

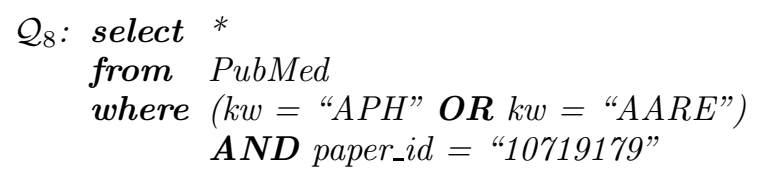

That is, by using both mapping tables, we are forced to restrict the identifiers of the retrieved articles. Since no restriction is imposed in the article_id attribute of the MedLine retrieved articles, a similar reasoning should be followed when translating this query for the PubMed articles. This reasoning supports not using mapping table id2id, in Figure 1(d), in translating this query.

The proposed technique accepts as input a query $q$ over a peer $P$ and a set of mapping tables $M$ from $P$ to a second peer $P^{\prime}$, and it uses the set $M$ to compute a complete translation $q^{\prime}$ of $q$. Initially, the algorithm converts $q$ into its equivalent disjunctive normal form. Then, it proceeds by considering each disjunct $D_{j}$ of $q$ in isolation. For each disjunct $D_{j}$, it selects a table for the translation, if this table's local attributes participate in an atom of the disjunct. Call $M_{j}$ the set of selected mapping tables. If the set of attributes of $D_{j}$ is contained in the set of attributes of $M_{j}\left(M_{j}\right.$ is relevant to $\left.D_{j}\right)$, the algorithm combines the mapping tables in $M_{j}$ into a single mapping table $m_{j}$ by using the $\wedge$-operator [11]. For the time being assume that $m_{j}$ mentions all the attributes exposed by peers $P$ and $P^{\prime}$. Then, the algorithm considers $D_{j}$ as a T-query $T_{j}$ containing only one row and computes a sound and complete translation of $T_{j}$ with respect to mapping table $m_{j}$. Then the translated T-query is converted into an equivalent relational algebra expression whose selection formula becomes a disjunct in the resulting query $q^{\prime}$.

Example 19 By using the algorithm described here, query $\mathcal{Q}_{1}$ is translated to query $\mathcal{Q}_{2}$ which is indeed its complete translation. As another example, assume that $\mathcal{Q}_{9}$ is a query retrieving information from MedLine about articles that mention protein $\mathrm{OPH}$ and were published in 1999:

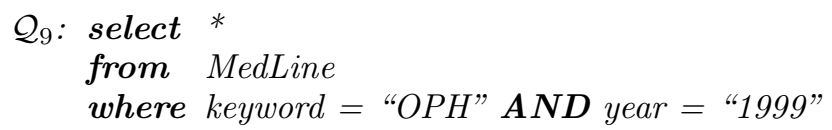

Then, our algorithm selects only mapping tables year2py and keyword2kw for the translation, resulting in the following query:

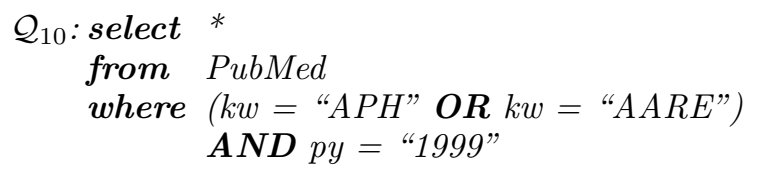

If any of the mapping tables $m_{j}$ computed by the above algorithm does not mention all the attributes exposed by $P$ and $P^{\prime}$, then it is extended to a mapping table $m_{j}^{\prime}$ that maps the extra attributes to any value. For example, mapping table ML2PM in Figure 3 is the extension of the following mapping table 


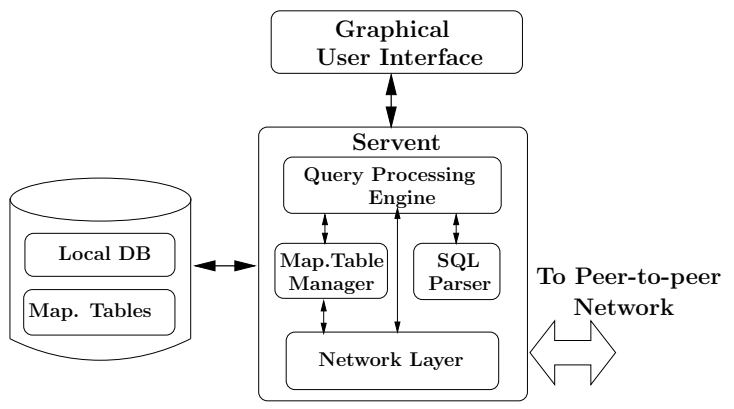

Figure 5: The architecture of a peer

\begin{tabular}{|c||c|}
\hline keyword & $k w$ \\
\hline OPH & APH \\
OPH & AARE \\
\hline
\end{tabular}

to the set of attributes \{article_id, keyword, year, paper_id, $k w, p y, p m\}$.

\section{Implementation}

We implemented our ideas over the prototype implementation of the Hyperion peer-to-peer data management system [4]. The structure of each peer in this system is shown in Figure 5. We provide a graphical user interface through which a user can set up acquaintances with new peers or pose local queries. Each peer in the system manages its own collection of data and it autonomously chooses a logical design and physical organization for the data. We use the MySQL relational DBMS to store both the peer data and any possible mapping tables that each peer might maintain.

The servent, which is the main component of this architecture, consists of four main modules. From these four modules, the query processing engine is the main focus of this work. It includes the implementation of the algorithms that convert SQL queries to their disjunctive normal form; convert SQL queries to their equivalent T-queries and back; and compute the complete translation of a query given a mapping table. Furthermore, it includes the algorithm for testing containment of T-queries and the algorithm to test whether a query is a sound translation of another one, with respect to a given mapping table. Finally, we also implemented the algorithm that, given a query $q$ and a set of mapping tables $M$, selects the set of mapping tables to use in order to compute a complete translation.

We implemented a number of optimizations to improve the efficiency of our algorithms. One such optimization relies on the fact that our representation of queries as T-queries allows us to store in the database both the query itself and the relationship with its sound translations. As an example, consider again the stored translation shown in Figure
4. Assume that the calculation of this translation happened some time in the past, but the system stores this relationship between the two T-queries in the database. Now, assume that a new query is issued on MedLine asking only for articles that mention protein $\mathrm{OPH}$ and were published in 1998. At this point, we could run the optimized version of the algorithm for computing complete translations in order to retrieve the corresponding PubMed articles. Alternatively, one can use the algorithm for Tquery containment to conclude that the correspondence between T-queries in Figure 4 can be used to compute the translation. In more detail, we test whether the T-query representation of the current query is contained in the left part of the table in the figure. Since this is the case in our running example, we treat the table in the figure as a mapping table and we use it to compute the translation of the current query. In this example, this computation will result in the selection of the right parts from the first two tuples.

An interesting application of the previous optimization relies on the observation that in peer-topeer systems users are often satisfied with answers that are not complete, as long as they are given the guarantee that anything that is retrieved is correct. With this in mind, even a query that retrieves all articles in MedLine mentioning proteins $\mathrm{OPH}$ or NGF receptor can be answered satisfactorily from the PubMed peer by just retrieving articles that mention these proteins and were published in some particular time interval (for example, the last 5 years). Testing for T-query containment is also central in this approach since the stored query is contained in the query being posed.

We conclude our overview by noting that the implementation of the query processing engine was done in the $\mathrm{C}$ programming language and it contains approximately four thousand lines of source code.

\section{Experiments}

To evaluate our algorithms, we undertook two studies. The objective of our first study is to investigate the performance of our translation algorithms with respect to three problem parameters, namely, the size of the input query, the size of the output query, and the size of the mapping tables used in the translation. The second study investigates the performance of our algorithms under large query load and examines the benefits of storing and re-using past translations. Due to lack of space, our second study is only available in the extended version of the paper [10]. The data used by both studies are real and are extracted from publicly available sources. We use these data to create distinct peers, one per 


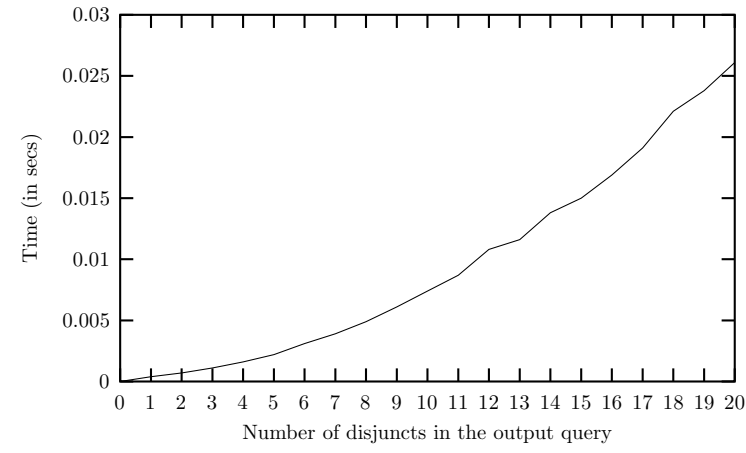

Figure 6: Generating large output queries

machine within the same LAN, and each peer has the structure shown in Figure 5.

\subsection{Biological Databases}

The data for this study belong to the MedLine, PubMed and SwissProt [2] databases. We populated each of the MedLine and PubMed relations with approximately 25000 tuples corresponding to proteinrelated articles. We assumed that MedLine articles are indexed, through the keyword attribute, by the currently approved protein name while PubMed articles are indexed by aliases of the approved names. Mapping table $i d 2 i d$ had approximately 24000 tuples while mapping table keyword2kw had approximately 12000 tuples. The former table maps an MedLine article to at most one PubMed article while the latter table maps currently approved protein names to their corresponding aliases. Both mapping tables were retrieved from SwissProt.

Given query $\mathcal{Q}_{1}$ and mapping table keyword2kw, we expect that the time to translate $\mathcal{Q}_{1}$ is influenced by the number of values that $\mathrm{OPH}$ is associated with, since this number influences the number of disjuncts, and thus the size, of the output query. Thus, the objective of our first experiment is to investigate the exact relationship between the time to perform a query translation and the size, in terms of disjuncts, of the translated query. For this purpose, we select 20 distinct input queries each of which is similar, in spirit, to query $\mathcal{Q}_{1}$, i.e., it retrieves MedLine articles for a particular keyword/protein. The queries were selected in such a manner that the first query refers to a protein with a single alias in keyword2kw, the second query refers to a protein with 2 aliases, and so on, while the last query refers to a protein with 20 aliases. Figure 6 shows the translation times (in seconds) for each of these queries. As we can see, the translation time scales gracefully and, even for large output queries, it is still fractions of a second.

In the previous experiment, all the input queries have only a single disjunct. In this experiment, we

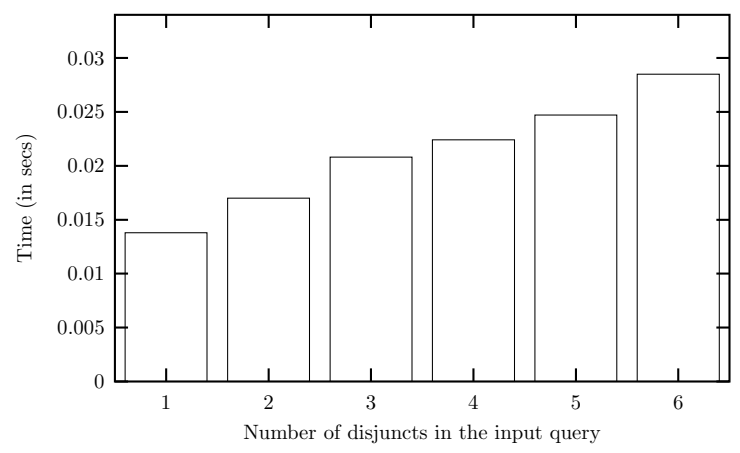

Figure 7: Translating large input queries

vary the number of disjuncts in the input query and we investigate how this influences the translation time. We start by selecting 20 distinct input queries each of which, once translated, results in an output query with 14 disjuncts. From the 20 input queries, 4 queries have 2 disjuncts, 4 have 3 disjuncts, and so on, and the last 4 queries have 6 disjuncts. We consider 4 alternative queries for the same number of disjuncts since, given the number of disjuncts in a query, there are different combinations with which these disjuncts can contribute to result in 14 output disjuncts. For example, for a query with just two disjuncts, each of the two input disjuncts can be translated to 7 output disjuncts, or alternatively, the first input disjunct can result in 10 output disjuncts while the second input disjunct can result in the remaining 4. In Figure 7, we average the translation times of input queries with the same number of disjuncts and we also report, in the first column, the translation of an input query with a single disjunct. Notice that as the number of disjuncts in the input query increases, there is a corresponding increase in the translation time of the query. Although there is a correlation between these two quantities, our next experiment shows that there is another factor that also comes into play during the translation process.

For this experiment, we use the input query that had the worst performance, in terms of time, in our previous experiment. This is the input query with 6 disjuncts, denoted with $D_{1}$ to $D_{6}$, where disjunct $D_{1}$, once translated, results in 9 output disjuncts while the remaining 5 input disjuncts all result in a single output disjunct. Notice again that the number of disjuncts in the output query is 14 . During this experiment, we translate this input query 6 times and the only difference between the translations is the order with which we translated the 6 input disjuncts. In particular, in the first run, the input disjunct $D_{1}$ is considered first for translation, while in the second run, disjunct $D_{1}$ is considered second in order for translation. Continuing in this fashion, in the sixth run, the five single-output disjuncts are 


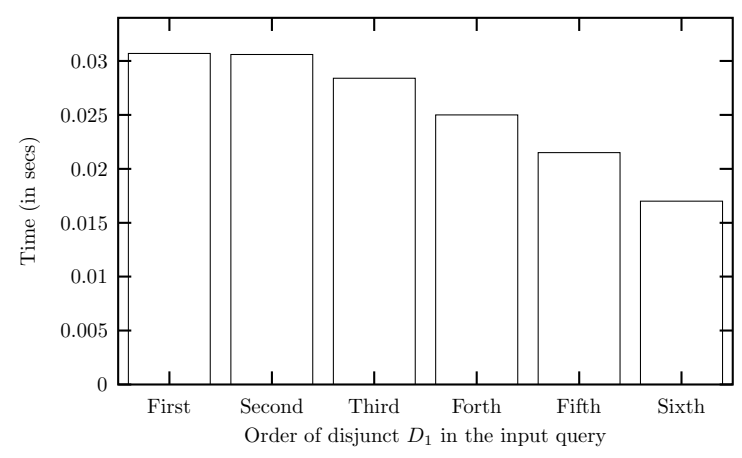

Figure 8: Translating a large input query

considered first, while disjunct $D_{1}$ is considered last. Figure 8 show the translation time for each of the six runs of the algorithm. The drop in translation time is due to the following reason. After a disjunct of the input query is translated, the algorithm checks whether any of generated output disjuncts is already part of the output query due to a previously translated input disjunct. The objective of this check is to avoid duplicate disjuncts in the output query, or pairs of disjuncts where one is contained in another. However, the check generates more comparisons, and thus more computation, if a large number of output disjuncts is generated early in the translation process. Hence, delaying the translation of a disjunct like $D_{1}$ causes reduced translation times. In general, reordering of input disjuncts seems beneficial and it can be achieved by storing frequency information about the values of a mapping table. Using these frequencies we can estimate the number of output disjuncts for each input disjunct.

We also experimented with varying the mapping table sizes. Our experiments show that this parameter does not influence the translation time. This is because we do not scan the whole table in order to locate the tuples to be used in the translation, but we use in-memory hash indexes. Our implementation of the hash indexes is customized to the semantics of mapping tables and thus takes into consideration the existence of variables in the tables.

\section{Conclusions}

We have considered the problem of data sharing between autonomous data sources. We used mapping tables to associate data from different sources and we have shown how the tables can be used in the translation of structured queries. We introduced the notions of sound and complete translations and we proposed algorithms to compute such translations and an algorithm to test if a query is a sound translation of another. We implemented our algorithms and we have presented experiments which show that these can be used in practice.

Our future work investigates algorithm optimizations along with support for more expressive query languages that include, for example, negation.

\section{References}

[1] PubMed/MedLine. http://www.ncbi.nlm.nig.gov/entrez/.

[2] SwissProt. http://www.ebi.ac.uk/swissprot/.

[3] S. Abiteboul, R. Hull, and V. Vianu. Foundations of Databases. Addison-Wesley, 1995.

[4] M. Arenas, V. Kantere, A. Kementsietsidis, I. Kiringa, R. J. Miller, and J. Mylopoulos. The Hyperion Project: From Data Integration to Data Coordination. SIGMOD Record, 32(3):53-58, 2003.

[5] C.-C. K. Chang and H. Garcia-Molina. Mind your vocabulary: Query mapping across heterogeneous information sources. In SIGMOD, pages 335-346, 1999.

[6] R. Fagin, P. Kolaitis, R. Miller, and L. Popa. Data exchange: Semantics and query answering. In ICDT, pages 207-224, 2003.

[7] A. Halevy, Z. Ives, D. Suciu, and I. Tatarinov. Schema Mediation in Peer Data Management Systems. In $I C D E, 2003$.

[8] M. Harren, J. M. Hellerstein, R. Huebsch, B. T. Loo, S. Shenker, and I. Stoica. Complex Queries in DHT-Based P2P Networks. In IPTPS, 2002.

[9] R. Huebsch, J. M. Hellerstein, N. L. Boon, T. Loo, S. Shenker, and I. Stoica. Querying the internet with pier. In $V L D B, 2003$.

[10] A. Kementsietsidis and M. Arenas. Data sharing through query translation in autonomous sources. Technical Report CSRG-491, CS Dept., University of Toronto, 2004.

[11] A. Kementsietsidis, M. Arenas, and R. J. Miller. Data mapping in p2p systems: Semantics and algorithmic issues. In SIGMOD, pages 325-336, 2003.

[12] M. Lenzerini. Data Integration: A Theoretical Perspective. In PODS, pages 233-246, 2002.

[13] J. W. Lloyd. Foundations of logic programming. Springer-Verlag New York, Inc., 1987.

[14] J. Madhavan and A. Y. Halevy. Composing Mappings Among Data Sources. In $V L D B$, pages $572-$ 583, 2003.

[15] W. S. Ng, B. C. Ooi, K. L. Tan, and A. Y. Zhou. Peerdb: A p2p-based system for distributed data sharing. In $I C D E$, pages 633-644, 2003.

[16] E. Rahm and P. A. Bernstein. On Matching Schemas Automatically. The VLDB Journal, 10(4):334-350, Dec. 2001.

[17] Y. Sagiv and M. Yannakakis. Equivalences Among Relational Expressions with the Union and Difference Operators. JACM, 27(4):633-655, 1980. 